\title{
Pulseoximetry is reliable for screening of sleep apnea regardless of software
}

\author{
Andreo Larsen* \\ Department of Clinical Neurophysiology and Diacor Medical Centre, University of Helsinki, Helsinki, Finland
}

\begin{abstract}
Because of the need for a cost-efficient method for screening of OSAS, a common and serious condition, this study compared two critical respiratory variables from the same data analyzed by two different software in seventy patients suspected of OSAS. There was an excellent correlation between AHI values derived from polysomnography and ODI values from simultaneous oximetry, regardless of the software used to analyze the data. The results indicate that home pulseoximetry offers a worthy and reliable option for screening of sleep apnea.
\end{abstract}

Abbreviations: OSAS: Obstructive sleep apnea syndrome; AHI: Apnea-hypopnea index; ODI: Oxygen desaturation index; PSG: Polysomnography; SaO2: Arterial oxygen saturation; SD: Standard deviation

\section{Introduction}

Obstructive sleep apnea syndrome (OSAS) is a common and serious condition [1-3]. At least $17 \%$ of middle aged men and $9 \%$ of women have sleep apnea [4] and even higher prevalence values have been published [5]. With such a high frequency there is need for a costeffective method for screening of OSAS.

The gold standard for diagnosis of OSAS is polysomnography (PSG). However, it is labor-intensive and expensive and therefore poorly suitable for screening purposes. Pulse oximetry was suggested for sleep apnea screening already in 1991 [6], but conflicting opinions have since then also been expressed $[7,8]$.

This study examines the applicability of pulse oximetry for screening of OSAS. With different equipment for oximetry available today, a special attention is given to the effect of the analysis software.

\section{Patients and methods}

The data was collected from the recordings of seventy consecutive patients (44 men, 26 women, mean age 50,2 \pm 12,3 years, BMI 29,3 \pm 5,6), who were referred to Diacor Medical Centre in Helsinki for an overnight home PSG. The recording was done with Nox T3 equipment (Nox Medical, Reykjavik, Island). The recorded parameters included flow, respiratory inductance plethysmography (RIP) with two belts, position, movement, ECG and pulse oximetry. The equipment includes a Nonin 3150 WristOx2 pulse oximeter (Nonin Medical, Plymouth, MN) for oximetry. The Nonin was programmed to sample oximetry data every 4 seconds and stores the data independently in its own memory, as well as transmitting it via Bluetooth to Nox. When the recordings were completed, the oximetry data was first extracted and analyzed with the nVision-software (Nonin Medical). The PSG data (including oximetry) was analyzed with Nocturnal software (Nox Medical). The start and stop times were identical. All data was verified by an experienced physician. From the gathered data the parameters used for this study are the apnea-hypopnea-index (AHI, number of apneas and hypopneas per hour of sleep) and ODI3 (hourly index of oxygen desaturations of $3 \%$ or more) as defined by the American Association of Sleep Medicine [9].

\section{Results}

The AHI varied from 1,1 to 105 with a mean of 23,0 (SD 24,0). The ODI3, when analyzed with Noxturnal software varied from 0,4 to 106 with a mean of 20,0 (SD 21,6). When analyzed with nVision software the ODI3 was from 0,5-106 with a mean of 22,2 (SD 21,6). There was an excellent correlation $(r=0,99)$ between the Nonin oximetry and AHI (and ODI) values from Nocturnal. In this material there were no false negative findings (i.e. ODI $3<5$ with AHI $>5$ ). Figure shows the correlation between AHI (Noxturnal) and ODI3 (nVision).

\section{Discussion}

The findings in this study demonstrated that overnight home oximetry alone, analyzed with nVision, gave ODI values which correspond very closely to AHI-values derived from state-of-the-art PSG recordings. This study thus confirms the findings of Vazquez, et al. [10] who found that "oximetry signal provides a close estimate of AHI as well as excellent diagnostic sensitivity and specificity for OSA", as well as those of Romem, et al. [11], who noted that "utilization of the photoplethysmographic signal enables accurate diagnosis of obstructive sleep apnea among a diverse cohort of patients". Also, Series, et al. [12], studying 50 patients with chronic heart failure, found that "overnight home oximetry is a reliable tool to screen for the presence of SRBDs”. Hang, et al. [13] showed overnight pulse oximetry

Correspondence to: Andreo Larsen, Department of Clinical Neurophysiology and Diacor Medical Centre, University of Helsinki, Helsinki, Finland, E-mail: andreo.larsen@fimnet.fi

Key words: oximetry, obstructive sleep apnea syndrome, apnea-hypopnea index, screening, cost-efficiency

Received: March 20, 2018; Accepted: March 26, 2018; Published: March 28 2018 


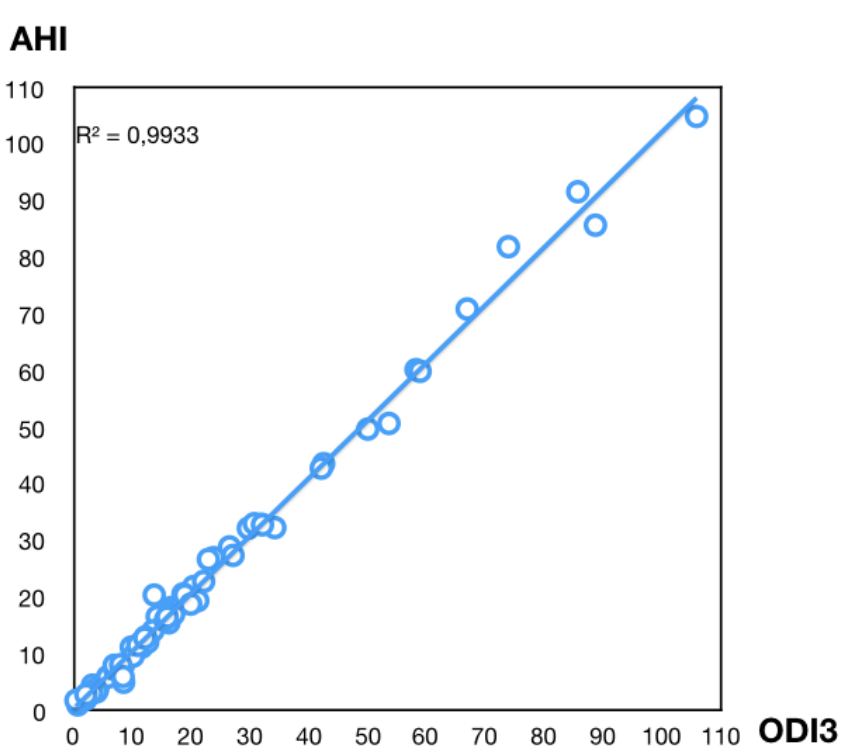

Figure 1. Correlation between Oxygen Desaturation Index (ODI3) analyzed with nVision software and simultaneously recorded Apnea-Hypopnea Index (AHI) analyzed with Noxturnal software in seventy patients with suspected sleep apnea

to have good sensitivity and selectivity for diagnosing patients with moderate and severe OSAS. Niijima, et al. [14] showed the usefulness of oximetry in sleep apnea screening also in occupational medicine.

Contrary to these findings Herer, et al. [7] claimed that "nocturnal oximetry taken alone may not accurately predict the presence or absence of obstructive sleep apnea". They used Ohmeda, Minolta and Nihon-Kohden oximeters and recorded mean and minimal $\mathrm{SaO} 2$ and time spent below $80 \%$ and $90 \% \mathrm{SaO} 2$ and concluded that "none of these individual variables had a satisfactory diagnostic value for the diagnosis of OSA". However, they did not record ODI which, as shown in this study, is a vital variable to record from oximetry.

Hussain, et al. stated that "oximetry did not help discriminate between those patients with or without OSAH" [8]. They did record the ODI2, ODI 3 and ODI 4 ( $2 \%, 3 \%$ and $4 \%$ desaturation indexes, respectively), but they used an Ohmeda oximeter, which stored the lowest $\mathrm{SaO} 2$ value once every 12 seconds, which is far too infrequently to adequately detect all significant desaturations, since the limit for apneas and desaturations is 10 seconds. This erroneous sampling frequency can explain their failure to detect many of the apnea patients.

In this study the ODI-values derived with different software were not fully identical, which is explained by the greater facility with the Nocturnal software to discard uncertain or borderline events. However, the difference was minimal and not significant.

The prevalence of OSAS is now known to be much higher $[4,5]$ than thought earlier [15]. This causes a necessity for cost-efficient methods for screening. The severity of OSAS is estimated on the basis of clinical data (excessive daytime sleepiness), AHI and oxygen saturation ( $\mathrm{SaO} 2)$ [16]. Since oximetry can give a reliable estimate of AHI as well as measuring the oxygen saturation, oximetry is suitable for screening of
OSAS. Being a simple and easy procedure, home oximetry has a good patient acceptance and costs less than PSG. It thus also has the ability of lighten the economic burden for public health.

In this study the oximetry and PSG data were verified by an experienced physician. Relying solely on automatic analysis may not be sufficient and is therefore discouraged.

\section{Competing interests}

The author has no competing interests.

\section{References}

1. Punjabi NM, Caffo BS, Goodwin JL, Gottlieb DJ, Newman AB, et al. (2009) Sleepdisordered breathing and mortality: a prospective cohort study. PLoS Med 6: e1000132. [Crossref]

2. Gottlieb DJ, Yenokyan G, Newman AB, O'Connor GT, Punjabi NM, et al. (2010) Prospective study of obstructive sleep apnea and incident coronary heart disease and heart failure: the sleep heart health study. Circulation 122: 352-360. [Crossref]

3. Redline S1, Yenokyan G, Gottlieb DJ, Shahar E, O'Connor GT, et al. (2010) Obstructive sleep apnea hypopnea and incident stroke; the Sleep Heart Health Study. Am J Respir Crit Care Med 182: 269-277. [Crossref]

4. Peppard PE, Young T, Barnet JH, Palta M, Hagen EW, et al. (2013) Increased prevalence of sleep-disordered breathing in adults. Am J Epidemiol 177: 1006-1014. [Crossref]

5. Heinzer R, Vat S, Marques-Vidal P, Marti-Soler H, Andries D, et al. Prevalence of sleep-disordered breathing in the general population: the HypnoLaus study. Lancet Respir Med 3: 310-318. [Crossref]

6. Williams AJ, Yu G, Santiago S, Stein M (1991) Screening for sleep apnea using pulse oximetry and a clinical score. Chest 100: 631-635. [Crossref]

7. Herer B, Roche N, Carton M, Roig C, Poujol V, et al. (1999) Value of clinical, functional, and oximetric data for the prediction of obstructive sleep apnea in obese patients. Chest 116: 1537-1544. [Crossref]

8. Hussain SF, Fleetham JA (2003) Overnight home oximetry: can it identify patients with obstructive sleep apnea-hypopnea who have minimal daytime sleepiness? Respir Med 97: 537-540. [Crossref]

9. AASM (2014) International classification of sleep disorders, ICSD-3. 3. ed. Darien IL: American Academy of Sleep Medicine.

10. Vázquez JC, Tsai WH, Flemons WW, Masuda A, Brant R, et al. (2000) Automated analysis of digital oximetry in the diagnosis of obstructive sleep apnoea. Thorax 55: 302-307. [Crossref]

11. Romem A, Romem A, Koldobskiy D, Scharf SM (2014) Diagnosis of obstructive sleep apnea using pulse oximeter derived photoplethysmographic signals. J Clin Sleep Med 10(3): 285-290. [Crossref]

12. Sériès F, Kimoff RJ, Morrison D, Leblanc MH, Smilovitch M, et al. (2005) Prospective evaluation of nocturnal oximetry for detection of sleep-related breathing disturbances in patients with chronic heart failure. Chest 127: 1507-1514. [Crossref]

13. Hang LW, Wang HL, Chen JH, Hsu JC, Lin HH, et al. (2015) Validation of overnight oximetry to diagnose patients with moderate to severe obstructive sleep apnea. $B M C$ Pulm Med 15: 24-23. [Crossref]

14. Niijima K, Enta K, Hori H, Sashihara S, Mizoue T, et al. (2007) The usefulness of sleep apnea syndrome screening using a portable pulse oximeter in the workplace. $J$ Occup Health 49: 1-8. [Crossref]

15. Young T, Palta M, Dempsey J ym (1993) The occurrence of sleep-disordered breathing among middle-aged adults. $N$ Engl J Med 328: 1230-1235. [Crossref]

16. Institute for Clinical Systems Improvement (ICSI). Diagnosis and treatment of obstructive sleep apnea. Bloomington (MN). March 2005.

Copyright: (C2018 Larsen A. This is an open-access article distributed under the terms of the Creative Commons Attribution License, which permits unrestricted use, distribution, and reproduction in any medium, provided the original author and source are credited. 